\title{
Composite MOFs as adsorbents for solid phase extraction combined with high performance liquid chromatography for the determination of polycyclic aromatic hydrocarbons in water
}

\author{
Yu Li ${ }^{1,2}$, Xiaojing Yang ${ }^{1,2}$ and Baohui $\mathrm{Li}^{12^{2 *}}$ \\ ${ }^{1}$ Department of Environment Science and Engineering, North China Electric Power University, Baoding, Hebei, 071003, China \\ ${ }^{2}$ A Hebei Key Lab of Power Plant Flue Gas Multi-Pollutants Control, Baoding, 071003, China
}

\begin{abstract}
Metal-organic frameworks (MOFs) are a kind of porous materials, which has large specific surface area, high porosity, diverse structure, excellent thermal stability and solvent stability etc. So, it has attracted extensive attention as an adsorbent for solid phase extraction (SPE). Polycyclic aromatic hydrocarbons (PAHs) are a kind of persistent organic pollutants that can lead serious hurts to the ecological environment and human health. The purpose of this paper is to optimize the types and proportion of mixed lanthanum based MOFs, and to improve the enrichment efficiency of SPE. Then a method to analysis PAHs by SPE-HPLC was established. Several main factors that could effected the pre-treatment efficient, such as the amount of adsorbent, the amount and $\mathrm{pH}$ of PAHs water sample, and the volume of eluent, were investigated. After optimized, the extraction of seven PAHs in water sample was achieved and the recovery rates were $80.67 \%, 80.06 \%, 54.69 \%, 82.62 \%, 118.87 \%, 70.30 \%$ and $95.57 \%$ respectively.
\end{abstract}

\section{Introduction}

PAHs are a kind of strong carcinogens and are susceptible to bio-accumulation [1]. Increased human production activities have result in increased levels of PAHs in various environmental media, including the air, surface and ground water [2]. Since PAHs concentration in all kinds of environments is too low to detected by many popular analytical instruments. Therefore, a sample pre-treatment is necessary before chromatographic separation of PAHs in the environment, such as SPE, solid phase micro-extraction (SPME), cloud point extraction (CPE). SPE is the most popular method for the enrichment of various analytes in a variety of matrices, including biological, industrial, environmental and food samples $[3,4]$.

SPE technology was developed in the 1980s and proved to be the effective tool for the separation and purification of analytes [5]. The advantages of SPE include simplicity, flexibility, high selectivity, automation, and higher enrichment factors [6]. MOFs are a kind of porous three-dimension materials with the advantages of high porosity and structural diversity. It is widely used in gas storage [7], catalysis [8], separation [9], drug delivery [10], chemical sensing [11,12], supercapacitors $[13,14]$, bio-medical imaging [15]. Due to the above advantages, as adsorbents for SPE, MOFs has gained widely attention.

Here, a method for the analysis of PAHs by SPE-HPLC was built, while lanthanum-terephthalic acid and lanthanum-trimesic acid as mixed adsorbents of SPE.

\section{Experimental}

\subsection{Instrument}

LC7011 binary pump and LC7020 UV/Vis detector (Hanon, China) were used for the high performance liquid chromatographic separation. ACCULAB analytical balance (Tianchuang, China) was employed to accurately weight PAHs standards.

\subsection{Chemicals and reagents}

The chemicals and reagents used are at least analytically grade. Lanthanum nitrate hexahydrate, methanol, dichloromethane and acetonitrile were purchased from Tianjin Kemiou Chemical Co., Ltd (Tianjin, China). Terephthalic acid, trimesic acid, naphthalene, acenaphthylene, acenaphthene, phenanthrene, anthracene, fluoranthene, and pyrene were purchased from Shanghai Dibai Chemical Technology co., Ltd (Shanghai, China). A mixed solution of seven PAHs of $200 \mathrm{mg} / \mathrm{L}$ was prepared as a stock solution. PAHs water sample was prepared by diluting the stock solution.

\subsection{The preparation of MOFs}

$4.505 \mathrm{~g}$ lanthanum nitrate hexahydrate and $0.864 \mathrm{~g}$ terephthalic acid, which weighted by analytical balance were mixed and dissolved in $15 \mathrm{~mL}$ of high-purity water

${ }^{\text {*Corresponding author's e-mail: baohuilee@gmail.com }}$ 
in a 25 beaker to prepare lanthanum- terephthalic acid MOFs. After magnetic stirring for $20 \mathrm{~min}$, the obtained substance was poured into a $40 \mathrm{~mL}$ Teflon-lined stainless autoclaves and maintained at $220^{\circ} \mathrm{C}$ for $72 \mathrm{~h}$. Then, the substance was washed with high-purity water and collected by filter paper. At last, the prepared MOFs was dried at $120^{\circ} \mathrm{C}$ for $12 \mathrm{~h}$. The process of preparation of lanthanum-trimesic acid is same to the previous one, except that $1.050 \mathrm{~g}$ lanthanum nitrate hexahydrate and $2.165 \mathrm{~g}$ trimesic acid were used as reaction reagents.

\section{Results and discussion}

\subsection{Determination of the proportion and flow rate of the mobile phase}

It is well known that the proportion and flow rate of the mobile phase have a great effect on the separation of the liquid chromatography. In this work, the effect of acetonitrile proportion in the mobile phase varied from $55 \%$ to $85 \%$ and the flow rate changed $0.8 \mathrm{~mL} / \mathrm{min}$ to 1.2 $\mathrm{mL} / \mathrm{min}$ on separation of PAHs were studied while acetonitrile-water as mobile phase. In Figure 1, it can be seen that with the acetonitrile proportion in mobile phase

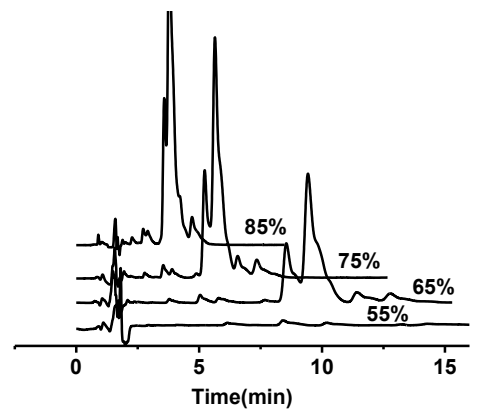

Figure 1. The different mobile phase proportion

\subsection{Effect of adsorbent mass on PAHs enrichment}

The proportion of lanthanum-terephthalic acid MOFs and lanthanum-trimesic acid MOFs were 1:1 in mixed MOFs. The effects of the mass of mixed MOFs on PAHs enrichment were studied. The experimental results are

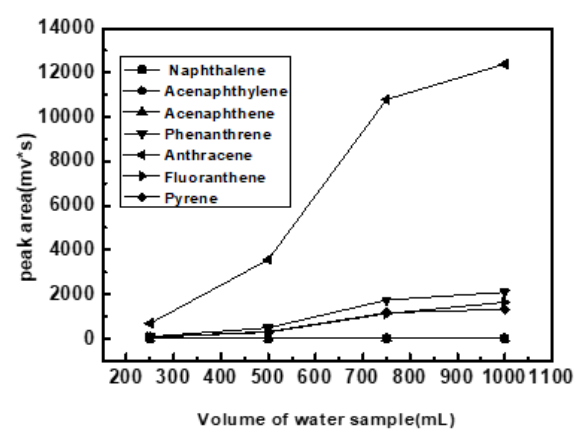

Figure 3. Effect of water sample amount on PAHs enrichment changed from $55 \%$ to $75 \%$, the resolution of seven PAHs were gradually increased, and the baseline separation was achieved when the acetonitrile proportion was $75 \%$. When the acetonitrile proportion in mobile phase was further increased, the resolutions of PAHs decreased. Therefore, $75 \%$ acetonitrile was chosen for subsequent experiments. $1.0 \mathrm{~mL} / \mathrm{min}$ flow rate was chosen as the compromise between retention time and peak area.

\subsection{Effect of water sample volume on PAHs enrichment}

In order to obtain the optimal volume of water sample, the effect of water sample volume on PAHs enrichment was studied. In Figure 3, it can be seen that while water sample volume varying from $250 \mathrm{~mL}$ to $500 \mathrm{~mL}$, the peak area of all seven PAHs had little change. However, while water sample volume varying from $500 \mathrm{~mL}$ to $750 \mathrm{~mL}$, the peak area increased significantly, exceeding $750 \mathrm{~mL}$, the peak area remained basically unchanged. Although the larger of the water sample amount, the larger of the peak area, however, the extraction time will be longer. Therefore, it was $750 \mathrm{~mL}$ that used as the optimal water sample volume.

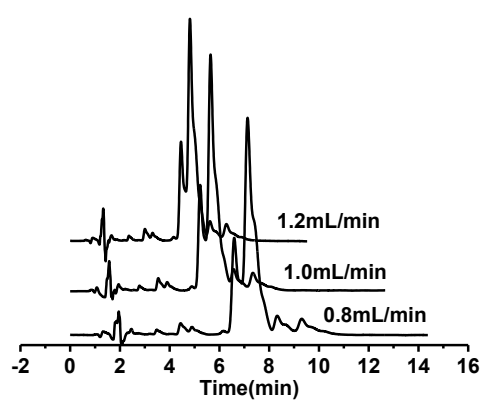

Figure 2. The different mobile rate

shown in Figure 4. As we can see that with the amount of the adsorbent increasing from $4 \mathrm{mg}$ to $8 \mathrm{mg}$, the peak area also increased. While the mass of adsorbent exceeding $8 \mathrm{mg}$, the peak area began to decrease. In fact, more amount adsorbent would lead to the longer extraction time. Therefore, $8 \mathrm{mg}$ of adsorbent was chosen for subsequent examines.

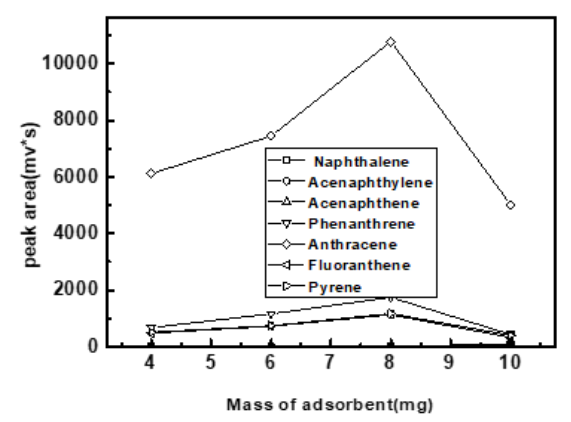

Figure 4. Effect of adsorbent mass on PAHs enrichment 


\subsection{Effect of pH on PAHs enrichment}

The effect of water samples with different $\mathrm{pH}$ values on the enrichment of PAHs was examined. The experimental results are show in Figure 5. With the $\mathrm{pH}$ of water sample increasing from 2.0 to 7.0 , the peak area increased. After that, peak area decreased. Therefore, a neutral water sample for subsequent experiments was selected.

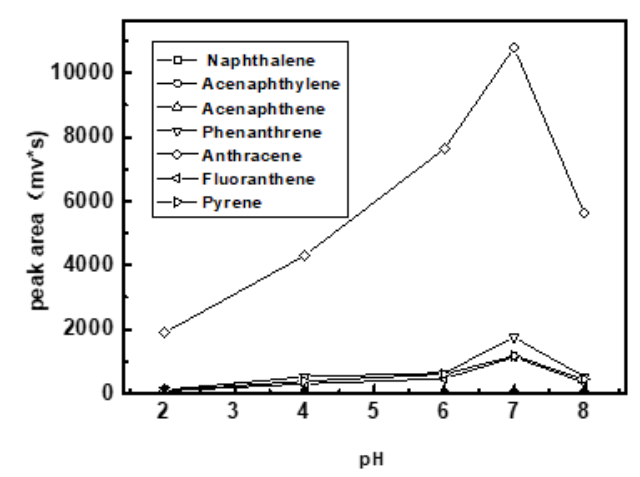

Figure 5. Effect of $\mathrm{pH}$ on PAHs enrichment

\subsection{Analytical Figures of Merit}

Different concentrations of PAHs water samples were prepared. Under the optimal conditions $(750 \mathrm{~mL}$ neutral water sample , $8 \mathrm{mg}$ adsorbent mass, and $0.8 \mathrm{~mL}$

\subsection{Effect of methanol elution volume on PAHs enrichment}

In order to determine the optimal methanol elution volume, the effect of methanol elution volume on PAHs was discussed. The experimental results are shown in Figure 6. While the amount of methanol increased from $0.4 \mathrm{~mL}$ to $0.8 \mathrm{~mL}$, the peak area was gradually increased. Then, the volume of methanol eluted was continuously increased, the peak area was gradually decreased with the elution volume varied from $0.8 \mathrm{~mL}$ to $1.2 \mathrm{~mL}$. Therefore, it was $0.8 \mathrm{~mL}$ that used as the optimal methanol elution volumes.

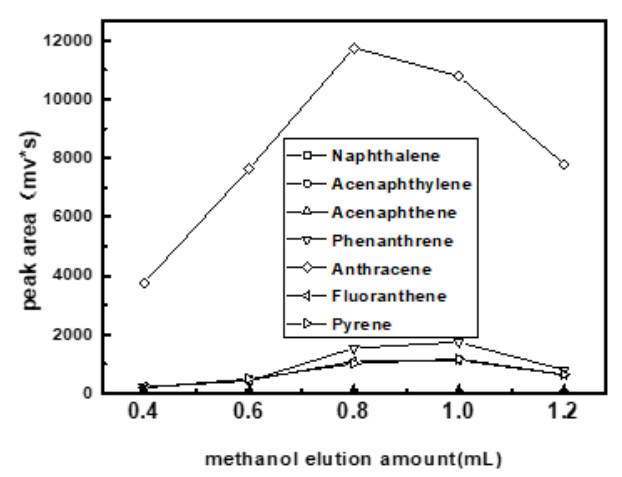

Figure 6. Effect of methanol elution amount on PAHs enrichment

methanol elution volume) PAHs in water samples were detected by SPE-HPLC. The experimental results are shown in Table 1.

Table 1. Recovery rate of seven polycyclic aromatic hydrocarbons.

\begin{tabular}{llll}
\hline Analyte & Linear equation & Correlation coefficient & Recovery rate $(\%)$ \\
\hline naphthalene & $\mathrm{y}=278.5 \mathrm{x}-8.6743$ & 0.999 & 80.67 \\
acenaphthylene & $\mathrm{y}=302.39 \mathrm{x}+0.8013$ & 0.991 & 80.06 \\
acenaphthene & $\mathrm{y}=663.98 \mathrm{x}-12.613$ & 0.9601 & 54.69 \\
phenanthrene & $\mathrm{y}=85375 \mathrm{x}-2160.5$ & 0.976 & 82.62 \\
anthracene & $\mathrm{y}=226254 \mathrm{x}-2233.9$ & 0.9817 & 118.87 \\
fluoranthene & $\mathrm{y}=49088 \mathrm{x}-850.01$ & 0.9581 & 70.30 \\
pyrene & $\mathrm{y}=41558 \mathrm{x}-855.22$ & 0.9776 & 95.57 \\
\hline
\end{tabular}

\section{Conclusion}

In summary, a method for the detected of trace PAHs in environmental samples by a mixed adsorbent of lanthanum-terephthalic acid and lanthanum-trimesic acid for SPE combined with HPLC was developed. The experimental results showed that the feasibility of using lanthanum-terephthalic acid and lanthanum-trimesic acid as composite adsorbents for the enrichment of trace PAHs in environmental sample.

\section{References}

1. Qin, N., He, W., Kong, X.Z., Liu, W.X., He, Q.S., Yang, B., Ouyang, H.L., Wang, Q.M., Xu, F.L.
(2013) Atmospheric partitioning and the air-water exchange of polycyclic aromatic hydrocarbons in a large shallow Chinese lake (Lake Chaohu). Chemosphere, 93: 1685-93.

2. Qin, N., He, W., Kong, X.Z., Liu, W.X., He, Q.S., Yang, B., Ouyang, H.L.,Wang, Q.M., Yang, C., Jiang, Y.J., Jorgensen, S.E., Xu, F.L., Zhao, X.L. (2014) Distribution, partitioning and sources of polycyclic aromatic hydrocarbons in the water-SPM-sediment system of Lake Chaohu, China. Sci. Total. Environ., 496: 414-23.

3. Wierucka, M., Biziuk, M. (2014) Application of magnetic nanoparticles for magnetic solid-phase extraction in preparing biological, environmental and food samples. Trac-Trend Anal. Chem., 71: 50-58. 
4. Souza Silva, E.A., Jiang, R.F., Rodriguez Lafuente, A., Gionfriddo, E., Pawliszyn, J. (2015) A critical review of the state of the art of solid-phase microextraction of complex matrices I. Environmental analysis. Trac-Trend Anal. Chem., 59: 224-235.

5. Erger, C., Schmidt, T.C. (2014) Disk-based solid-phase extraction analysis of organic substances in water. Trac-Trend Anal. Chem., 61: 74-82.

6. Zwir Ferenc, A., Biziuk, M. (2006) Solid phase extraction technique-Trends, opportunities and applications. Pol. J. Environ. Stud., 15: 677-90.

7. Dinca, M., Long, J R. (2008) Hydrogen storage in microporous metal-organic frameworks with exposed metal sites. Angew. Chem. Int. Edit., 47: 6766-6779.

8. Ma, L.Q., Abney, C., Lin, W.B. (2009) Enantioselective catalysis with homochiral metal-organic frameworks. Chem. Soc. Rev., 38: 1248-1256.

9. Li, J.R., Kuppler, R.J., Zhou, H.C. (2009) Selective gas adsorption and separation in metal-organic frameworks. Chem. Soc. Rev., 38: 1477-1504.

10. Cui, Y.J., Yue, Y.F., Qian, G.D., Chen, B.L. (2012) Luminescent Functional Metal-Organic Frameworks. Chem. Rev., 112: 1126-1162.

11. Yi, F.Y., Chen, D X., Wu, M.K., Han, L., Jiang, H.L. (2016) Chemical Sensors Based on Metal-Organic Frameworks. Chempluschem, 81: 675-690.

12. Kumar, P., Deep, A., Kim, K.H. (2015) Metal organic frameworks for sensing applications. Trac-Trends in Analytical. Chemistry., 73: 39-53.

13. Wang, H.L., Zhu, Q.L., Zou, R.Q., Xu, Q. (2017) Metal-Organic Frameworks for Energy Applications. Chem, 2: 52-80.

14. Sheberla, D., Bachman, J C., Elias, J.S., Sun, C.J., Shao Horn, Y., Dinca, M. (2017) Conductive MOF electrodes for stable supercapacitors with high areal capacitance. Nat. Mater., 16: 220-224.

15. Della Rocca, J., Liu, D., Lin, W. (2011) Nanoscale metal-organic frameworks for biomedical imaging and drug delivery, Acc. Chem. Res., 44: 957-968. 